\title{
CUIDAR E INVESTIGAR: DESAFIOS METODOLOGICOS EN ENFERMERÍA
}

\author{
Martha Lucia Vásquez Truisi ${ }^{1}$
}

${ }^{1}$ Ph.D. Profesora Titular de la Escuela de Enfermería de la Universidad del Valle. Colombia. E-mail: maluvasq@gmail.com

RESUMEN: A pesar del pluralismo metodológico en la investigación del cuidado en enfermería, existen algunos desafíos que deben considerarse para continuar el desarrollo y el fortalecimiento del cuerpo de conocimientos de la disciplina. En este artículo se señalan algunos como, transitar de estudios mono disciplinares a inter y trans disciplinares, desarrollar propuestas investigativas como práctica rutinaria con sentido esencialmente transformador que mejoren la calidad del cuidado cotidiano en los servicios de salud, y aprender a rescatar las experiencias de cuidado para sistematizarlas generando, a partir de este proceso, conocimiento. Todos estos desafíos deben constituir ejercicios rigurosos que busquen dar mayor validez a las investigaciones que sobre el cuidado se desarrollan para crear y mantener una sólida base científica que promueva mejores resultados en los cuidados de las personas y que refleje, no sólo, el ejercicio de una práctica fundamentada científicamente sino además, el desarrollo de una profesión autónoma.

DESCRIPTORES: Enfermería. Cuidado de enfermería. Métodos de investigación.

\section{CARE AND RESEARCH: METHODOLOGICAL CHALLENGES IN NURSING}

\begin{abstract}
In spite of the methodological pluralism in research on nursing care, there are some challenges that should be considered to continue developing and enhancing the body of knowledge of the discipline. This article points to some of these like: going from mono-disciplinary to inter- and trans-disciplinary studies, developing research proposals as a routine practice with an essentially transforming that improve the quality of everyday care in health care services, and learning to gather nursing care experiences to systematize them and, thereby, generate knowledge through this process. All these challenges should constitute rigorous exercises seeking to give greater validity to the research being conducted on health care to create and maintain a sound scientific basis, which promotes better results in caring for individuals and which not merely reflects the exercise of a scientifically based practice, but also the development of an autonomous profession.
\end{abstract}

DESCRIPTORS: Nursing. Nursing care. Research methods.

\section{CUIDAR E PESQUISAR: DESAFIOS METODOLÓGICOS NA ENFERMAGEM}

\begin{abstract}
RESUMO: Apesar do pluralismo metodológico na pesquisa do cuidado em enfermagem, há alguns desafios a serem considerados para continuar o desenvolvimento e o fortalecimento do corpo de conhecimentos da disciplina. Este artigo reflete sobre os desafios de transitar de estudos monodisciplinares à estudos inter e transdisciplinares, desenvolver projetos de pesquisa como prática cotidiana com a intensionalidade de transformar para melhor a qualidade do cuidado nos serviços de saúde e aprender a resgatar as experiências de cuidado a fim de sistematizá-las de maneira a gerar conhecimento a partir deste processo. O enfrentamento destes desafios exige rigor no desenvolvimento das propostas de modo a buscar maior validade das investigações sobre o cuidado, criar e manter uma sólida base científica que promova melhores resultados dos cuidados às pessoas e que reflita , não somente, o exercício de uma prática com bases científicas mas também o desenvolvimento de uma profissão autônoma.
\end{abstract}

DESCRITORES: Enfermagem. Cuidado de enfermagem. Métodos de pesquisa. 


\section{INTRODUCCIÓN}

Actualmente, podemos advertir que a pesar de haber un gran pluralismo metodológico en la investigación del cuidado en Enfermería, existen algunos desafíos que deben considerarse para continuar el desarrollo y el fortalecimiento del cuerpo de conocimientos de la disciplina. En este artículo, se señalan algunos caminos metodológicos cualitativos y cuantitativos, entre los cuales se destacan la sistematización de experiencias, la meta-síntesis, el meta-análisis, la integración y complementariedad de los paradigmas cualitativo y cuantitativo y el trabajo inter y trans disciplinario entre otros. Todos ellos pueden constituir ejercicios rigurosos de análisis, interpretación e integración de resultados que buscan dar mayor validez a las investigaciones que sobre el cuidado se desarrollan y de esa manera contribuir, de manera significativa, a generar conocimiento para crear y mantener una sólida base científica para la práctica que promueva mejores resultados en los cuidados de las personas y que refleje, no solo, el ejercicio de una práctica fundamentada científicamente sino además el desarrollo de una profesión autónoma.

\section{Cuidar e investigar: consideraciones para avanzar en enfermería}

Antes de abordar los desafíos metodológicos relativos a la investigación del cuidado para la enfermería y para la salud es necesario recordar las premisas que Ann Davis ${ }^{1}$ expone sobre lo que es el cuidado desde dos posturas: La primera de ellas entendiendo el acto de cuidar como brindar o proporcionar cuidados y la segunda postura como una respuesta emocional del cuidador hacia la persona que cuida.

Davis ${ }^{1}$ señala que el primer significado de cuidar se ha orientado a realizar actividades para otras personas y con otras personas que se creen son beneficiosas para ellas; de esta forma, los cuidados a menudo han implicado e implican al menos a dos personas cuya relación está determinada principalmente por la responsabilidad que tiene una de ellas, generalmente la enfermera, de dar respuesta a otra persona y de satisfacer sus necesidades en salud. Como es de suponer, este tipo de relación generalmente se da entre personas desconocidas que a menudo, no sólo no comparten su historia, sino que el contexto de donde proviene cada una ellas es diferente.

La segunda postura es relativa al cuidado y la cual ha cobrado vigor en las últimas dos décadas, es aquella en la cual el cuidador ejerce el cuidado como una respuesta emocional que conlleva una preocupación por el otro y da importancia a la relación, al afecto, a la franqueza y a la atención de las necesidades de la persona a la que se cuida. Diversos autores desde la disciplina de la enfermería ${ }^{2-4}$ y otros, de otras disciplinas ${ }^{5}$, han ahondado en la urgencia y en la relevancia de destacar el cuidado humano como un acto de solidaridad y de interés genuino por el otro. Esto, como es conocido por todos nosotros, no puede ser sólo un discurso elocuente sino que debe concretarse en acciones "cuidadoras" para las cuales, no sólo es necesario poner toda la atención y esfuerzo, sino que además se debe ser capaz de generar confianza, serenidad, seguridad y apoyo efectivo al otro lo cual, a la luz de los contextos sociales que vivimos, es difícil de realizar.

Estas dos posturas nos hacen pensar que cuidar, per se, no es una simple tarea y como tal, en el sentido estricto, no puede medirse en los procesos investigativos, ya que cuidar abarca tanto el aspecto instrumental como el aspecto existencial o expresivo. Esta reflexión evidencia una de las complejidades que subyacen en el proceso de investigación del cuidado pues estamos hablando de abordar al ser humano como unidad.

Adicionalmente, la investigación del cuidado no puede sustraerse de los rasgos sustantivos que deben caracterizar los sistemas de salud, por lo cual se deben realizar los esfuerzos necesarios para considerar principios como la universalidad, la calidad, la equidad y contención de costos, aspectos que, evidentemente, hacen que estos procesos de investigación sean más complejos.

Igualmente, tampoco debería sustraerse del tipo de demanda que actualmente se tiene para los servicios esto es, una demanda de atención de cobertura limitada, tardía, poli medicamentosa, tecnológicamente dependiente, que requiere de especialistas de segundo y tercer nivel de atención y de un seguimiento casi constante. Como es obvio, se trata de una atención de alto costo, para las instituciones y para los pacientes y el cuidado de enfermería no puede abstraerse a esa realidad. ${ }^{6}$.

Concomitante con este panorama que se cierne sobre la atención en salud, el Consejo Internacional de Enfermeras ${ }^{7}$ ha precisado que el futuro de la enfermería debe tener en cuenta que el cuidado tendrá especial énfasis en los adultos mayores, en el cuidado en casa y, desafortunadamente por el dolor que para las personas implica, los profesionales de enfermería tendrán que prepararse para 
ofrecer el cuidado en contextos multiculturales de violencia, conflicto y desastres actuando bajo el lema "Pensar globalmente y actuar localmente".

De otro lado, las directrices actuales para avanzar en el mejoramiento de la salud de las personas se orienta a privilegiar la promoción y la prevención, desarrollando acciones enfocadas al diagnóstico y detección oportuna de enfermedades crónico-degenerativas, estimulando el uso óptimo de los servicios de salud de primer nivel e impulsando actividades tendientes a modificar los estilos de vida.

Este panorama dificulta mas la elección de metodologías de investigación para la indagación del cuidado y desafía abiertamente a nuestra disciplina para buscar y profundizar en estrategias que nos ayuden a resolver las cuestiones relativas al mismo.

Ahora bien, sabemos que para la disciplina de enfermería es indispensable generar conocimiento para crear y mantener una sólida base científica para la práctica, que promueva mejores resultados en los cuidados de las personas sujeto de cuidado, y que refleje, no sólo, el ejercicio de una práctica fundamentada científicamente sino además el desarrollo de una profesión autónoma.

Por ello, el deber y sentido de continuar avanzando en la búsqueda y generación de conocimiento de cuidar como esencia ontológica de la enfermería y en ese sentido, explicar y demostrar, la realidad del fenómeno; buscar relaciones, generalizar y validar lo relativo al cuidar, es una tarea permanente y comprometida que las enfermeras debemos tener por el sentido mismo que esta labor entraña.

Esta tarea investigativa se ha venido cumpliendo utilizando diferentes caminos y abordajes teniendo claro que no existe uno que sea lo suficientemente amplio como para dar explicación a todos los aspectos que se manejan en la práctica del cuidado; en ese sentido, el camino en la evolución de nuestro conocimiento disciplinar ha sido fructífero desde la época de Florence Nightingale en la cual el foco de investigación se centró en el qué hacer, para luego pasar al dominio del hacer técnico es decir, al cómo hacer, pasando luego al por qué hacer, hasta nuestros días en los cuales se ha venido profundizando en comprender cuál es el saber propio de nuestra disciplina ${ }^{8}$ incorporando dentro del mismo el diseño y creación de nuevas maneras de cuidar para mejorar la calidad de vida. ${ }^{9}$

Igualmente importante es referir lo que el Consejo Internacional de Enfermeras ha declara- do importante para el despuntar de este siglo en lo relativo con la investigación en enfermería ${ }^{10}$ : la generación de conocimiento disciplinar para enfermería; el análisis de los aspectos relacionados con la prestación de servicios de salud y la calidad del cuidado; el estudio del impacto de las intervenciones de enfermería en las metas de salud y bienestar y la integración de la práctica basada en la evidencia. Todo este abanico de necesidades para dinamizar y ampliar los horizontes de cuidado, desde escenarios distintos y promover el trabajo en equipo entre las instituciones formadoras del talento humano y las prestadoras de los servicios de salud .

Ahora bien, evidenciamos que los temas referentes al cuidado son complejos, que aun, a pesar de los esfuerzos que se han venido haciendo por parte de las enfermeras, el camino por allanar es grande y todavía hay una gran brecha frente a las áreas propuestas y la investigación desarrollada.

Veamos, a grosso modo, cuales han sido los abordajes que con mayor énfasis se han seguido en la investigación en enfermería. En a nivel mundial se utilizaban metodologías experimental/cuasiexperimental, evaluativas, estudios correlacionales, epidemiológicos, investigación cualitativa especialmente fenomenológica y la aplicación de sistemas. ${ }^{11}$ Adicionalmente, en estudio sobre los coloquios de investigación 2000-2006, los autores encontraron que los diseños más utilizados en la presentación de los trabajos correspondía a la metodología cuantitativa (86\%) lo que da cuenta de la tradición positivista de la disciplina en las Américas, pudiendo observarse también que los trabajos descriptivos son dominantes (96\%). ${ }^{12}$ Durante este periodo la investigación cualitativa inicia un desarrollo incipiente correspondiendo a un $14 \%$ del total. Esta situación muestra que el desarrollo disciplinar es lento ya que el crecimiento teórico debe ser sustentado por producciones basadas en aportes realizados por investigaciones explicativas y comprensivas. Adicionalmente, la mayoría de los trabajos de las autoras corresponden a estudios mono disciplinares.

El breve recuento presentado, muestra un panorama sobre las metodologías que ha venido desarrollando la enfermería, particularmente en nuestro contexto latinoamericano, el cual, si bien da cuenta del avance y de la multiplicidad metodológica que ha estado ocurriendo desde la mitad del siglo pasado, nos invita a identificar algunos retos que debemos enfrentar para mejorar nuestra practica investigativa. 
A continuación presento seis desafíos metodológicos que deberíamos afrontar con el propósito de continuar el avance y profundización del estudio de la acción de cuidar para la disciplina de enfermería.

\section{Desafío 1: transitar de estudios mono disciplinares a inter $y$ trans disciplinares}

Si nos aproximamos al ser humano, no con una concepción fragmentaria, sino como unidad, como ser integral, es necesario que tengamos en cuenta que el cuidado es un proceso complejo en el que intervienen diferentes actores en un contexto económico social y en un momento histórico determinado. Podríamos decir que de esa manera, la realidad del cuidado se muestra de forma poliédrica con contradicciones en un contexto espacio-temporal. Igualmente, sabemos que hay interdependencia con otras disciplinas y que los instrumentos y las formas de construir teoría no solamente han sido generadas por la disciplina de la Enfermería sino que es patrimonio de otras disciplinas Esta realidad representa un desafío, pues la investigación exigiría un abordaje menos reduccionista y fragmentario y mas interrelacionado con otras disciplinas en un complejo esfuerzo metodológico, pues las diferentes disciplinas muchas veces implican diversos abordajes y maneras de pensar el fenómeno. Este esfuerzo implica un proceso y una filosofía de trabajo que entra en acción a la hora de enfrentar los problemas y cuestiones que preocupan cuando se estudia el cuidado. ${ }^{13}$.

La investigación debe contemplar diferentes planos de comprensión, obedeciendo a las especificidades de los variados problemas y situaciones de salud. En el plano metodológico, se reconoce el avance en el desarrollo de técnicas y métodos cuantitativos facilitados enormemente por la informática.

Pero las intensas polémicas que han generado las acciones resultantes de estos análisis, al tiempo que señalan los actuales límites de dichos métodos marcan la necesidad de continuar el perfeccionamiento de su metodología, además de reclamar una mejor articulación con las ciencias sociales y económicas, buscando entender mejor el fenómeno del cuidado. Pero para que esa interdependencia sea una meta alcanzable se hace necesario una revisión al sistema de formación de los profesionales en enfermería, para que desde la misma formación, las enfermeras estén más comprometidas con la realidad del cuidado y su transformación.
Debemos entrar en la vertiente de la reforma del pensamiento, avanzando en un método trans disciplinario, capaz de ligar las áreas del conocimiento y trabajar la multi dimensionalidad de los fenómenos ya que la manera de ver las cosas actualmente sólo permite visiones parciales, unilaterales y cerradas lo que limita apreciar lo complejo de todo. ${ }^{14}$

\section{Desafío 2: Aprender y desarrollar propuestas investigativas como práctica rutinaria con sentido esencialmente transformador que mejoren la calidad del cuidado cotidiano en los servicios de salud}

Sabemos que hay un distanciamiento entre la teoría que se imparte en las instituciones formadoras del talento humano y la práctica que se ejerce en cada escenario del cuidado. Esta ruptura, se hace evidente también entre los resultados que se obtienen de las investigaciones primarias realizadas por las enfermeras y los pocos cambios que han permitido en el cotidiano del cuidado. ${ }^{15}$ De allí que se hace necesario que los profesionales de enfermería se involucren y participen activamente en procesos de investigación que les permita identificar los problemas de la practica y resolverlos mediante dichos procesos.

Este desafío implica que los profesionales de enfermería integren, como una necesidad a su práctica laboral, ejercicios de investigación científica. Como esta labor no se ha asumido completamente como práctica rutinaria, ello ha impedido perfilar acciones desde esa lógica. El reto de investigar desde el espacio profesional cotidiano implica el desarrollo de metodologías que, sin dejar de lado el rigor que debe caracterizarlas, deben facilitar la celeridad del proceso especialmente para descubrir, no sólo aquellos elementos que condicionan el problema, sino también como un procedimiento para evaluar el cambio de la situación que originó la acción de cuidado. Por tanto, ya que no hay recetas y por fortuna hay multiplicidad de posibilidades metodológicas, es necesario tener claros los criterios por los cuales vamos a identificar, entre otros, qué metodología utilizar, qué pasos dar y cuáles técnicas usar.

En esta tarea, el acompañamiento de las instituciones formadoras del talento humano en enfermería es vital. El intercambio y cooperación han demostrado ser una poderosa estrategia para alcanzar objetivos y metas comunes. Realizar esta tarea conjunta redundará, sin lugar a dudas, en que las enfermeras asistenciales se motiven, 
valoren y consuman críticamente los resultados de otras investigaciones; identifiquen problemas propios de su entorno y se animen a realizar cambios en su práctica de manera crítica. ${ }^{16} \mathrm{Ne}-$ cesitamos una ciencia con conciencia, en la cual, apliquemos lo descubierto, no de manera ciega o caprichosa a los intereses políticos o económicos sino teniendo en cuenta las necesidades de las personas a las que cuidamos.

\section{Desafío 3: A prender a rescatar las experiencias para sistematizarlas y a partir de este proceso generar conocimiento}

El significado más usado hace referencia al ordenamiento y clasificación de datos e informaciones, estructurando de manera precisa categorías, relaciones, posibilitando la constitución de bases de datos organizados. Pero a lo que aquí se refiere es de ir más allá de clasificar la información; se trata de mirar las experiencias como procesos históricos, procesos complejos en los que intervienen diferentes actores, que se realizan en un contexto económico-social determinado y en un momento institucional del cual formamos parte. Sistematizar experiencias significa entonces entender por qué ese proceso se está desarrollando de esa manera, entender e interpretar lo que está aconteciendo, a partir de un ordenamiento y reconstrucción de lo que ha sucedido en dicho proceso.

Por lo tanto, en la sistematización de experiencias, partimos de hacer una reconstrucción de lo sucedido y un ordenamiento de los distintos elementos objetivos y subjetivos que han intervenido en el proceso, para comprenderlo, interpretarlo y así aprender de nuestra propia práctica. ${ }^{17}$

El desafío está en no quedarnos sólo en la reconstrucción de lo que sucede sino pasar a realizar una interpretación crítica de lo sucedido para poder extraer lecciones aprendidas que tengan una utilidad para el futuro.

Por tanto, el reto en este aspecto es el que seamos capaces de pasar de lo descriptivo y narrativo a lo crítico e interpretativo. Esto no es fácil, pues no tenemos la costumbre de teorizar, no hemos desarrollado suficientemente nuestra capacidad analítica y muchas veces nuestra formación nos ha empujado a simplemente ser consumidores pasivos de los conocimientos de otras personas.

El punto clave de la sistematización, es el interrogar a la experiencia para entender por qué pasó lo que pasó, comprendiendo cómo se pusieron en juego los diferentes componentes y factores presentes en la experiencia, para poder enfrentarla ahora con una visión de transformación. En ese sentido, sistematizamos nuestras experiencias para aprender críticamente de ellas y así mejorar nuestra propia práctica de cuidado, compartir nuestros aprendizajes y para contribuir al enriquecimiento de la teoría.

De acuerdo a la literatura, no hay una sola manera de sistematizar experiencias. Podrían indicarse algunas posibilidades o la combinación de algunas de ellas: Desde los actores, en forma participativa, como una sistematización formal al concluir la experiencia, o como una sistematización que se hace sobre la marcha.

Esta metodología implica resolver dialécticamente la relación entre saber empírico y saber científico. Lamentablemente sufrimos un paradigma de ciencia que no valora el conocimiento empírico, el saber local y sólo pone énfasis en el conocimiento acumulado, en el saber constituido académicamente.

La sistematización de experiencias es una gran posibilidad para que se expresen y se desarrollen y se divulguen los conocimientos y saberes locales, que tienen mucho que aportar al enriquecimiento del pensamiento científico. Esto también permitirá asumir más creativamente nuestros proyectos, pues no tendremos una actitud pasiva que se reduce a aplicar los conocimientos y verdades que ya existen, sino que impulsaremos nuestra capacidad de crear conocimientos adecuados a la realidad que vivimos y sus particularidades.

\section{Desafío 4: trabajar en metodologías donde se propicie la integracióny complementariedad de los paradigmas cualitativo y cuantitativo}

Como es conocido por todos, los dos paradigmas se basan en una visión del mundo diferente pero complementaria; los dos, por tanto, son necesarios para obtener un conocimiento más claro y completo de la realidad. Aunque en algunas investigaciones en enfermería se han trabajado metodologías con los dos paradigmas, los esfuerzos aun son escasos en lo relativo al cuidado. Has ventajas el utilizar conjuntamente metodologías con los dos tipos de paradigmas para alcanzar objetivos múltiples, robustecer mutuamente los tipos de métodos y llevar a cabo la triangulación. ${ }^{18}$ Cada vez se percibe con más fuerza cómo la separación y dicotomía empobrece la investigación. Cuando se quiere comprender un fenómeno sólo desde una mirada se "limitan seriamente las posi- 
bilidades de la ciencia al alcanzar descripciones o cuantificaciones, comprensiones o explicaciones, criticas o legitimaciones válidas, precisas y fiables de la realidad social" ${ }^{19: 17-8}$

Lo importante no es acentuar las diferencias sino identificar los nexos de unión y la búsqueda de la complementariedad. La defensa de la complementariedad metodológica es proclamada na obra, "Los siete saberes necesarios a la educación del futuro", en donde el autor explica que la complejidad de los hechos o fenómenos, exigen la presencia de visiones distintas pero concurrentes, lo que ofrecerá una riqueza de matices que se pueden escapar a cada una de ellas de forma aislada. ${ }^{20}$

Al referirse a la investigación cualitativa, Bartolomé, subraya la finalidad de la investigación como elemento clave a tener en cuenta al indicar que "la investigación cualitativa ha alcanzado en esta década un reconocimiento explícito como vía, no única desde luego, para acceder al conocimiento científico pero no se vislumbra, al menos por ahora, la existencia de un sólo paradigma, ni siquiera circunscribiéndonos a la metodología cualitativa: más bien reconocemos un pluralismo de enfoques que son consecuencia, no tanto de las estrategias utilizadas para acercarse a la realidad (que son muy similares) ni de la concepción objeto de estudio (la realidad aparece como construida y tiende a abordarse desde una perspectiva holística) sino de la finalidad de la investigación (comprender, construir unas teorías, establecer relaciones consistentes; transformar la práctica....)". ${ }^{21: 15-6}$

La complementación e integración paradigmática y metodológica a la que se acaba de hacer mención es cada vez más habitual en la investigación en las Ciencias Sociales y Humanas. En la ciencia de la enfermería esta tendencia también se ha ido perfilando en un estilo de investigación en el que se integran distintos métodos en un mismo diseño, esto es la investigación multimétodo. La combinación de múltiples métodos, materiales empíricos, perspectivas, e incluso observadores, focalizados en un estudio singular, debe entenderse como una estrategia que agrega rigor, amplitud y profundidad al trabajo del investigador. ${ }^{22}$

Básicamente para enfermería son tres las razones que nos llevarían a la integración de distintos métodos en un mismo diseño de investigación: de un lado, contar con dos o más imágenes distintas del cuidado, realidad social o fenómeno objeto de estudio; de otro lado, compensar las limitaciones de un método con las fortalezas de otro y finalmente reforzar la validez de los resultados.
Estas razones se hallan en la base de las tres estrategias de integración denominadas estrategias de complementación, de combinación y triangulación, respectivamente. ${ }^{23}$ Las describimos brevemente:

Complementación: La finalidad de esta estrategia es llevar a cabo una investigación con dos estructuras metodológicas distintas, para conseguir como resultado último un informe síntesisinterpretativo de los resultados procedentes de cada método.

Combinación: Esta estrategia trata de integrar un método (sea cuantitativo o cualitativo) en el otro método, con el objeto de fortalecer la validez de este último, compensando sus limitaciones de tal forma que las fortalezas de uno de los métodos son utilizadas para compensar las debilidades del otro.

Triangulación: Con esta modalidad de integración se pretende reforzar la validez de los resultados. Es especialmente útil cuando lo que buscamos son resultados convergentes y válidos bajo la premisa de que cuanto más diferentes sean los métodos que muestran idénticos resultados mayor será la evidencia de su veracidad.

No quiero pasar por alto los problemas de legitimidad científica que plantea la investigación multi método. Son muchos los autores que participan en este debate y sostienen la tesis de que las posibilidades de integración sólo pueden ser resueltas en el plano metodológico, siempre y cuando la estructura resultante sea en sí misma coherente.

Al diseñar una investigación con los atributos del "estilo multi-método", debe entenderse que la integración debe aplicarse a la formulación del problema de investigación, a los objetivos, a la recolección de datos y al análisis e interpretación.

Es necesario considerar también, además de las situaciones habituales que se tienen cuando se realiza cualquier tipo de investigación, que el estudio multi-método enfrenta otro tipo de retos.

El primero, hace referencia al perfil formativo de los investigadores, pues al menos uno de los investigadores debe estar familiarizado con la aplicación de ambos tipos de orientaciones metodológicas.

El segundo reto de la práctica de la investigación multi-método es el aspecto relativo al tiempo puesto que esta dimensión en cada tipo de investigación y en cada una de sus fases es diferente. El resultado de integrar una investigación realizada por encuesta estructurada, con otra, de 
naturaleza etnográfica, puede ser incoherente, debido a que la rapidez de la primera se enfrenta con la lentitud de la segunda.

El tercer reto hace alusión al incremento económico que conlleva la realización de un estudio multi método.

\section{Desafío 5: Trascender lo local y particular del cuidado enriqueciéndolo con lo global y colectivo}

Los estudios comparativos o cross cultural son un excelente ejercicio para hacer esa transición. Usualmente se encuentran numerosos trabajos de investigación en los cuales se estudia el fenómeno del cuidado en una comunidad o subcultura en particular sin que se compare o identifique los aspectos similares o diferentes en las diferentes culturas. La invitación es que estos esfuerzos no queden sólo en lo local y especifico de la subcultura que se aborda. Con los estudios cross cultural, como generalmente se les llama, se identifican similitudes y diferencias en las maneras de abordar el cuidado en distintas culturas. Mediante esta investigación se pueden descubrir aspectos universales que ayudan a entender mejor el comportamiento humano en relación al cuidado humano.

La comparabilidad de los datos es un requisito fundamental para realizar investigaciones Cross cultural. Un aspecto central en este asunto es contar con o desarrollar instrumentos de medición que posean tanto validez como equivalencia. Este tipo de estudios a pesar de que son empresas complejas y difíciles de realizar por la inversión de tiempo y esfuerzo considerable que implican son necesarios pues a través de ellos se puede avanzar hacia la explicación o teorización respecto al porque de las diferencias encontradas en los diferentes entornos.

Desafío 6: Profundizar el conocimiento y visión del cuidado realizando metodologías de investigación basadas en la evidencia de enfermería

El volumen de investigaciones generadas en los últimos años y la necesidad de sintetizar la eclosión de resultados de innumerables investigaciones que se realizan han propiciado que se estructuren metodologías de investigación que sinteticen, resuman y revisen sistemáticamente la información obtenida en diferentes estudios sobre un problema determinado. Estas metodologías, relativamente nuevas, ocurren en los llamados Meta-análisis cuando se trata de investigaciones cuantitativas, o Meta-síntesis en caso de estudios cualitativos.

En su esencia, el Meta-análisis es una revisión bibliográfica, pero a diferencia del método clásico que ésta utiliza, consistente en comentar, analizar y comparar descriptivamente ensayos clínicos individuales, adicionalmente relaciona sistemáticamente y cuantifica gran diversidad de resultados y ofrece conclusiones cuantitativas y cualitativas sobre el aspecto estudiado. ${ }^{24}$ Adicionalmente, como la gran mayoría de los ensayos clínicos que se publican, tienen un tamaño muestral pequeño, al combinar estos limitados estudios se pueden llegar a conclusiones más confiables.

Vale la pena recalcar que esta metodología se aplica sólo a estudios empíricos, no teóricos, por tanto los resultados producidos son cuantitativos, en vez de cualitativos, los cuales a su vez son susceptibles de ser configurados en formas estadísticas comparables como en el caso de las correlaciones, proporciones, tamaños de efectos, entre otros.

Su utilidad, para el caso del estudio del cuidado en enfermería puede resultar restringida debido a que, por la esencia misma de este fenómeno, no resulta adecuado utilizar ensayos clínicos controlados que son precisamente los que se privilegian cuando de comparar y relacionar resultados sistemáticamente en el Meta-análisis se trata. A pesar de esta limitación, es importante que, desde y para enfermería, no se deje de lado este camino metodológico ya que en la literatura científica hay experiencias interesantes y rigurosas en las cuales, a través de estudios experimentales, se evalúan por ejemplo, aspectos relativos a la eficacia del cuidado y en ese sentido, dichos aspectos pueden ser objeto de análisis en algunos experimentos.

Como todo método de inferencia, el metaanálisis tiene sus problemas y limitaciones. Uno de los principales problemas es que al ser un estudio que generalmente se ejecuta de forma retrospectiva, tiene una posibilidad mayor de introducir sesgos. Otros problemas de importancia son los que se derivan del procedimiento de combinar datos de múltiples ensayos clínicos entre los cuales pudiera haber diferencias en cuanto a criterios utilizados. El llamado sesgo de publicación es otra gran fuente de errores pues existe la tendencia a creer que los ensayos clínicos realmente realizados son solamente los que han sido publicados. Muchos ensayos clínicos no son publicados por diversas causas, especialmente aquellos que no registran diferencias entre los distintos grupos de 
tratamiento. De este modo, los meta-análisis que incluyen sólo los resultados de ensayos clínicos publicados pueden tender a sobreestimar el efecto del tratamiento experimental.

El segundo tipo de estudios es la meta-síntesis el cual es un tipo de evidencia científica que consiste en un complejo ejercicio de interpretación que busca ampliar la relevancia y utilidad de los estudios cualitativos. Esta aproximación constituye "[...] un modelo crítico reflexivo de revisión, síntesis y difusión de evidencias; asimismo es un método de aprendizaje y ejercicio profesional para la toma de decisiones basadas en las evidencias útiles y pertinentes $[\ldots . .]^{\prime 2} .{ }^{25: 92-5}$

Sin importar la técnica específica o el método utilizado para desarrollarla, la meta-síntesis siempre busca una interpretación más comprehensiva de los fenómenos. La comparación constante, la interpretación y la integración de los hallazgos son pasos claves en este tipo de investigación, cualquiera que sea el método o técnica seleccionada; la diferencia entre las técnicas radica en el alcance o meta esperada que determina qué y cómo hacer la comparación, interpretación e integración en mención.

La meta-síntesis es entonces, una técnica a desarrollar para trascender del conocimiento particular y específico generado a partir de estudios cualitativos primarios, hacia una comprensión más amplia de los fenómenos a través del análisis e interpretación de los hallazgos de dichos estudios; de este modo, la meta-síntesis se constituye en una vía fundamental con la que se documentan evidencias útiles para la disciplina de enfermería que permiten su aplicación en la práctica del cuidado.

\section{Condiciones para lograr enfrentar los desafíos}

Enfrentar estos desafíos implica reconocer que algunas acciones deberían realizarse para dinamizar, transformar y transcender los procesos metodológicos en la investigación. En ese sentido, desde las aulas de las universidades es necesario que se continúe trabajando en propiciar un pensamiento crítico entre los alumnos, desarrollando competencias que les permitan formular preguntas, entender las ideas de otros, evaluar los argumentos que se ofrecen en favor de ellas y formular posiciones y fundamentarlas para promover una discusión razonada.

Sin duda alguna estos retos precisan que observemos cómo se aborda la formación académica para la investigación del cuidado de enfermería, e incluso asumir el reto de que el escenario la pe- dagogía de la investigación del cuidado no debe estar exento de una práctica fundamentada en el hallazgo científico producto del quehacer investigativo cotidiano.

Esta condición, desplegada de manera dinámica, permitirá que el futuro profesional de enfermería tenga conciencia y motivación investigativa. Se podría prever que uno de los resultados de esta estrategia seria el que las enfermeras, cuidadoras e investigadoras, exploren las situaciones emergentes del día día en la práctica del cuidar para elaborar sus problemas de estudio y analizar la mejor manera de abordar dichos problemas. Para ello las instituciones de formación de enfermería deben asumir la práctica de cuidar y de investigar como prácticas interdisciplinares complementarias y entrelazadas haciendo que la enfermera las incorpore como un hábito.

Otra estrategia prioritaria para enfrentar los desafíos metodológicos para la investigación del cuidado en enfermería consiste en promover y desarrollar redes de investigación entre profesionales de docencia y de servicio en donde se configuren espacios de discusión para el aprendizaje continuo y el apoyo entre colegas, lo que redundará en el desarrollo de la investigación disciplinar y colaborativa, así como propender por el desarrollo de la investigación inter y transdisciplinar.

Lo anterior implica no sólo, el compromiso de las instituciones de docencia y de servicio pues ellas deben estar dispuestas a destinar recursos y tiempo para ello, sino también la utilización de los medios tecnológicos de información para dinamizar el flujo y consecución de la producción académica e investigativa.

\section{CONCLUSIONES}

La investigación en enfermería esta llamada a ir elaborando procedimientos metodológicos que faciliten a los profesionales, elementos que contribuyan a liberarles de la rutina de hacer siempre lo mismo y del mismo modo.

Es necesario cuestionar lo que hacemos intentando contemplar la acción de cuidar sin minusvalorar la fundamentación teórica vigente sino perfeccionándola.

Los desafíos planteados, han tratado de poner en relieve algunas luces y sombras metodológicas que tiene la investigación del cuidado en enfermería. Las luces serán cada día más luminosas puesto que contamos con talento humano con gran inquietud por la búsqueda de la verdad 
e inquietud por transmitirla en el cuidado que prestan a las personas con el propósito final de contribuir en el mejoramiento de la calidad de vida de la sociedad.

\section{REFERENCIAS}

1. Davis A. Tenir cura i l'etica del tenir cura en el segle XXI: qué sabem i qué hem de qüestionar [online]. Barcelona (ES): Collegi oficial Infermeria de Barcelona; 2006 [acceso 2010 Jun]. Disponible en: http:/ /212.9.72.18/uploadBO/Noticia/Documents/ CONFER\%C3\%A8NCIA\%20ANNE\%20DAVIS\%20 CASTELL\%C3\%AO_DEF.PDF

2. Watson J. The theory of human caring: retrospective and prospective. Nurs Sci Q. 1997 Spr; 19(2):49-59.

3. Orem D.E. Nursing concepts of practice. $2^{\mathrm{a}}$ ed. New York (US): McGraw-Hill; 1980.

4. Leininger M. Transcultural nursing: concepts, theories and practices. New York (US): John Wiley; 1978.

5. Boff L. Saber cuidar: ética do humano - compaixão pela terra. $3^{\text {a }}$ ed. Petrópolis (RJ): Vozes; 1999.

6. Oulton J, Caldwell P. Nursing research: meeting today's health challenges perspectives from the international Council of Nurses. Global Forum Update on Research for Health. 2007; 4: 121-8.

7. Consejo Internacional de Enfermeras (CIE). Declaración de posición del CIE sobre investigación en enfermería. En: La investigación de enfermería, instrumento de acción. Genebra (CH): CIE; 1999.

8. Oliviera Gomes VL, Schubert VM, Coelho de Souza MI, Cezar Vaz MR. Evolución del conocimiento en la enfermería: del cuidado popular a la construcción de teorías. Invest. Educ Enferm. 2007 Sep; 15(2):108-15

9. Garzón N. Retos de enfermería para el nuevo milenio. In: Cuidado y practica de enfermería. Bogotá (CO): Unibiblos; 2000.

10. Consejo Internacional de Enfermeras. Investigación en enfermería: establecimiento de un programa internacional de Investigación. Ginebra Informe del Comité de expertos de Investigación de Enfermería. Genebra (CH): CIE; 1997.

11. Murrain E. Tendencias de la investigación en enfermería. Repert Med Cir. 2009; 18(2):90-6.
12. Cometto C, Piovan M, Gómez P. Aportes de los coloquios panamericanos a la investigación en enfermería periodo 2000-2006. Texto Contexto Enferm. 2008 Out-Dez; 17(4):720-6.

13. TorresSantomeJ.Globalizaçaoeinterdisciplinaridade: o currículo integrado. Porto Alegre (RS): Artmed; 1998.

14. Morin E. La cabeza bien puesta. $2^{\mathrm{a}}$ ed. Buenos Aires (AR): Nueva Visión; 2001.

15. Loayza Enríquez, Blanca Katiuzca. Buscando evidencias en la ciencia y el arte del cuidado de enfermería: retos y desafíos. Evidentia [online]. 2010 Abr-Jun [acceso 2010 Jul 29]; 7(30). Disponible en: http:// www.index-f.com/evidentia/n30/ev1530.php

16. Burns N, Grove S. Investigación en enfermería. $3^{a}$ ed. Madrid (ES): Elsevier; 2004.

17. Holliday OJ. Dilemas y desafios de la sistematizacion de experiencias [online]. En: Seminario ASOCAM: Agricultura Sostenible Campesina de Montaña. Cochabanba Bolivia (BO): Intercooperatio; 2001 [acceso 2010 Jul 7]. Disponible en: http://www. grupochorlavi.org/webchorlavi/sistematizacion/ oscarjara.PDF

18. Cook TD, Reichardt CH.S. Métodos cualitativos y cuantitativos en investigación cualitativa. Madrid (ES): Morata; 1986.

19. Bericat E. La integración de los métodos cuantitativos y cualitativos. Barcelona (ES): Ariel; 1998.

20. Morin E. Los siete saberes necesarios a la educación del futuro. Paris (FR): UNESCO; 1999.

21. Bartolomé M. Investigación cualitativa en educación ¿Comprender o transformar? Rev Investigación Educativa. 1992; 20: 7-36.

22. Denzin N, Lincoln Y, editors. Handbook of qualitative research. Thousand Oaks (US): Sage; 1994.

23. Bericat E. La integración de los métodos cuantitativos y cualitativos. Barcelona (ES): Ariel; 1998.

24. Laporte JR. Principios básicos de investigación clínica. Madrid (ES): Ergon; 1993.

25. Romero MN. Bases conceptuales de la enfermería basada en la evidencia. In: Anales de la IV Jornada Internacional de Investigación en Ciencias de la Enfermería, 2010 Jun 24-26, Chiclayo, Perú. Chiclayo (PE): Universidad Católica Santo Toribio de Mogrovejo; 2010. 